\title{
High glucose levels increase the expression of neurotrophic factors associated with p-p42/p44 MAPK in Schwann cells in vitro
}

\author{
HAO ZHU ${ }^{1}$, WEN-JUAN YU ${ }^{2}$, YAN LE $^{1}$, WEN-JIN WANG ${ }^{1}$, FENG LI $^{1}$, TING GUI ${ }^{1}$, \\ YUE-MING WANG ${ }^{1}$, WO-DONG SHI ${ }^{3}$, WEN-LONG DING ${ }^{1}$ and XIAN-QUN FAN ${ }^{3}$ \\ ${ }^{1}$ Department of Anatomy; ${ }^{2}$ Shanghai Mental Health Center, Shanghai Jiao Tong University School of Medicine; \\ ${ }^{3}$ Department of Ophthalmology, Shanghai Ninth People's Hospital, Shanghai Jiao Tong \\ University School of Medicine, Shanghai, P.R. China
}

Received January 11, 2012; Accepted April 12, 2012

DOI: $10.3892 / \mathrm{mmr} .2012 .896$

\begin{abstract}
Diabetic peripheral neuropathy (DPN) is one of the most common complications of diabetes mellitus. One contributing factor to DPN is altered neurotrophism due to changes in the synthesis and expression of neurotrophins. Schwann cells (SCs) are the myelin-forming cells of the peripheral nervous system that promote nerve regeneration through the expression and secretion of neurotrophic factors (NTFs). Therefore, in this study, using SCs cultured in the presence of high levels of glucose for $24 \mathrm{~h}$, with and without the p42/p44 mitogenactivated protein kinase (MAPK) inhibitor, PD98059, we investigated the effect of high glucose levels on SCs over a short period of time. The cultured cells were evaluated using 3(4,5-dimethylthiazol-2-yl)2,5-diphenyltetrazolium bromide (MTT) assay, Hoechst staining, immunocytochemistry, reverse transcriptase-polymerase chain reaction and western blot analysis. High glucose levels did not promote morphological abnormalities or decrease the viability of SCs. However, high glucose levels enhanced the expression of nerve growth factor (NGF) and brain-derived neurotrophic factor (BDNF) and induced the activation of p42/p44 MAPK in cultured SCs in a dose-dependent manner. Additionally, the phosphorylation
\end{abstract}

Correspondence to: Dr Wen-Long Ding, Department of Anatomy, Shanghai Jiao Tong University School of Medicine, No. 227 South Chongqing Road, Shanghai 200025, P.R. China

E-mail: dingw1500@sina.com

Dr Xian-Qun Fan, Department of Ophthalmology, Shanghai Ninth People's Hospital, Shanghai Jiao Tong University School of Medicine, No. 227 South Chongqing Road, Shanghai 200025, P.R. China E-mail: fanxq@sh163.net

Abbreviations: DPN, diabetic peripheral neuropathy; SC, Schwann cell; NTFs, neurotrophic factors; NGF, nerve growth factor; BDNF, brain-derived neurotrophic factor; FBS, fetal bovine serum; MAPK, mitogen-activated protein kinase

Key words: Schwann cell, high glucose, neurotrophic factors, p42/ p44 mitogen-activated protein kinase of p42/p44 MAPK may be associated with the expression of NTFs by SCs exposed to high glucose conditions; the excessive activation of p42/p44 MAPK inhibited the expression of NTFs. These observations demonstrate that exposure to high glucose levels lead to acutely elevated levels of NGF and BDNF in SCs over a short period of time, which may be involved in the $\mathrm{p} 42 /$ p44 MAPK pathway.

\section{Introduction}

Peripheral neuropathy is one of the most common complications of diabetes mellitus (1). The most prevalent form of diabetic peripheral neuropathy (DPN) develops as a distal symmetric sensorimotor neuropathy. Diabetic autonomic neuropathy may also accompany distal symmetric neuropathy and can disrupt a number of sympathetic or parasympathetic functions (2). Hyperglycemia and poor blood glucose control are precipitating events that initiate a series of complex and interrelated metabolic and vascular insults that underlie the etiology of diabetic neuropathy (3). One contributing factor to DPN is an altered neurotrophism resulting from changes in the synthesis and expression of neurotrophins that can directly act on distinct subpopulations of sensory and motor neurons (4). Schwann cells (SCs) are the myelin-forming cells of the peripheral nervous system, which are critical for regulating and maintaining myelin thickness (5) and for promoting nerve regeneration through the expression and secretion of neurotrophic factors (NTFs), such as the nerve growth factor (NGF) and brain-derived neurotrophic factor (BDNF).

However, much less attention has been focused on examining growth factors in hyperglycemically-stressed SCs. Although recently there has been some research on this aspect (6), only the expression of NTFs in SCs exposed to high glucose conditions for a long period of time or in models of diabetic neuropathy has been investigated. For short-term exposure to high glucose levels, the impact on the viability and NTF expression in SCs is not clear. Detecting this impact is critical in order to further clarify DPN pathogenesis. SC culture systems are useful for investigating these effects. In the present study, SCs were cultured under high glucose conditions for $24 \mathrm{~h}$ and cell morphology, viability, NTF expression and other signaling molecules were examined. 


\section{Materials and methods}

Primary cultures of SCs and high levels of glucose treatment. SC cultures were obtained using a previously described method (7). Cells were incubated in serum-free DMEM containing $30 \mathrm{mM}$ (control group), $60 \mathrm{mM}$ (Glu-60 group), $90 \mathrm{mM}$ (Glu-90 group) and $150 \mathrm{mM}$ (Glu-150 group) glucose or $30 \mathrm{mM}$ glucose $+120 \mathrm{mM} \mathrm{L}$-glucose (L-Glu120 group) for $24 \mathrm{~h}$. In addition, in a number of experiments, $50 \mathrm{mM}$ PD98059, a mitogen-activated protein kinase (MAPK) inhibitor, was added to the medium. In these experiments, the cells were grown in serum-free DMEM with $90 \mathrm{mM}$ glucose for $24 \mathrm{~h}$ following pre-treatment with PD98059 for $2 \mathrm{~h}$.

Immunofluorescence/Hoechst staining. Cells were fixed with $4 \%$ paraformaldehyde incubated with the rabbit polyclonal S-100 antibody (1:200, DAKO, Glostrup, Denmark) overnight at $4^{\circ} \mathrm{C}$ at a $1: 500$ dilution in PBS plus $1 \%$ bovine serum albumin and $0.1 \%$ Triton X-100. Cells were then incubated with FITC-conjugated goat anti-rabbit IgG (Cappel/ Organon Teknika Corp, Aurora, OH, USA). Samples were also co-stained with $10 \mu \mathrm{g} / \mathrm{ml}$ Hoechst 33342 (Sigma). Cells were mounted on slides using Biomedia Gel/Mount (Electron Microscopy Sciences, Hatfield, PA, USA), and slides were examined using a Leica DM2500 microscope with a CCD 2/3 camera.

MTT assay. The 3(4,5-dimethylthiazol-2-yl)2,5-diphenyltetrazolium bromide (MTT) assay was used to study SC viability. Cells were plated in a 96-well plate and cultured for $24 \mathrm{~h}$ under high glucose conditions. Then, $10 \mu \mathrm{l}$ of MTT stock solution $(10 \mathrm{mg} / \mathrm{ml}$; Sigma) were added and the cultures were incubated for another $4 \mathrm{~h}$ at $37^{\circ} \mathrm{C}$. Subsequently, the medium was discarded and insoluble dark blue formazan was dissolved in DMSO and quantified at $570 \mathrm{~nm}$ using a reference wavelength of $630 \mathrm{~nm}$ and a microtiter plate reader (Bio-Rad Laboratories, Hercules, CA, USA).

Reverse transcriptase-polymerase chain reaction (RT-PCR). Following $24 \mathrm{~h}$ of high glucose treatment, total RNA was extracted from the cells using TRIzol reagent (Invitrogen, Carlsbad, CA, USA). RNA was reverse-transcribed in a final volume of $20 \mu \mathrm{l}$, containing $1 \mu \mathrm{g}$ of total RNA, $50 \mathrm{pM}$ oligo(dT), $10 \mu \mathrm{M}$ deoxyribonucleoside triphosphate (dNTP), 20 units RNasin, 10 units AMV reverse transcriptase (Promega, Madison, WI, USA) and $4 \mu 1$ of $5 \mathrm{X}$ reverse transcriptase buffer containing DEPC $\mathrm{H}_{2} \mathrm{O}$ (Takara, Dalian, China) supplied by the manufacturer. PCR was performed using $2 \mu \mathrm{l}$ of synthesized cDNA with $0.125 \mu \mathrm{l}$ Taq polymerase (Takara), $2 \mu \mathrm{l}$ dNTPs, $0.5 \mu \mathrm{l}$ each primer, $2.5 \mu$ l PCR buffer, which was supplied by the manufacturer, and DEPC $\mathrm{H}_{2} \mathrm{O}$ was added to give a total reaction volume of $25 \mu \mathrm{l}$. All common components were added to a master mix and aliquoted. The sequences of the oligonucleotide primers were (5'-3'): NGF forward, TCCACCCACCCAG TCTTCCA and reverse, GCCTTCCTGCTGAGCACACA; BDNF forward, AGCTGAGCGTGTGTGACAGT and reverse, TCCATAGTAAGGGCCCGAAC; GAPDH forward, CACC ACCATGGAGAAGGCC and reverse, GATGGATGCCTT GGCCAGG. The cycling conditions were as follows: initial denaturation at $95^{\circ} \mathrm{C}$ for $5 \mathrm{~min}$ followed by 28 cycles of $95^{\circ} \mathrm{C}$ for $1 \mathrm{~min}, 55^{\circ} \mathrm{C}$ for $1 \mathrm{~min}, 72^{\circ} \mathrm{C}$ for $2 \mathrm{~min}$ and a final extension at $72^{\circ} \mathrm{C}$ for $5 \mathrm{~min}$. GAPDH was used as the loading control.

Western blot analysis. SCs were incubated with high levels of glucose for $24 \mathrm{~h}$, then washed with PBS and lysed with RIPA buffer (Sigma). The total protein concentration was then determined using a kit based on the bicinchoninic acid (BCA) method. For western blot analysis, $20 \mu \mathrm{g}$ of the cell extract were electrophoresed on a $12 \%$ SDS/polyacrylamide gel and transferred to a PVDF membrane. The membrane was blocked with 5\% non-fat dry milk in Tris-buffered saline plus $0.1 \%$ Tween-20 (TBS-T), and incubated overnight with anti-NGF, anti-BDNF (1:400; Santa Cruz Biotechnology, Santa Cruz, CA, USA), anti-phospho-p44/42 MAPK (p-ERK1/2), antip44/42 MAPK (ERK1/2; 1:1000; Cell Signalling Technology, MA, USA) and anti-GAPDH (1:2000, Sigma) antibodies. The membrane was washed and then incubated with the peroxidase-conjugated secondary antibody (1:1000, Sigma) in 5\% non-fat dry milk in TBS-T buffer for $1 \mathrm{~h}$. Detection was performed using an enhanced chemiluminescence (ECL) detection kit (Pierce, Rockford, IL, USA).

Statistical analysis. One-way ANOVA followed by a Newman-Keuls multiple comparison test was used to compare control and treated groups, and $\mathrm{P}<0.05$ was considered to indicate a statistically significant difference.

\section{Results}

High glucose conditions do not induce abnormal SC morphological characteristics. In order to examine the morphological characteristics of SCs, we used a polyclonal antibody for S-100 to immunostain the SCs (green) and Hoechst 33342 to stain the nuclei (blue). After SCs were exposed to increasing concentrations of glucose, there were no marked differences in the cell body size, process length and apoptosis rate in all groups (Fig. 1).

High glucose concentrations do not reduce SC viability. The viability of SCs was assessed using an MTT reduction assay. Cells were incubated with various concentrations of glucose for $24 \mathrm{~h}$. It was found that cell viability in the Glu-60 group was $103.4 \pm 9.0 \%$ compared to that of the control group and, at glucose concentrations up to $150 \mathrm{mM}$, it was $105.9 \pm 9.6 \%$ (Fig. 2). There was no statistical difference between any of the SC cultures. The findings demonstrated that high glucose levels had no effect on SC viability.

High glucose levels induce the upregulation of NTF $m R N A$ levels in SCs. NGF and BDNF mRNA levels were evaluated in SCs using RT-PCR. Compared to the control cells, the mRNA levels of NGF $(\mathrm{P}<0.05)$ and BDNF $(\mathrm{P}<0.01)$ in SCs were significantly increased at glucose concentrations ranging between $60-90 \mathrm{mM}$ (Fig. 3). Additionally, compared to the control cultures, the level of NGF mRNA was decreased in the SCs exposed to $150 \mathrm{mM}$ glucose $(\mathrm{P}<0.05)$, whereas there was no significant difference in the levels of BDNF mRNA (Fig. 3).

High glucose levels enhance the expression of SC-derived NTFs. To examine the expression of NTFs, we examined 


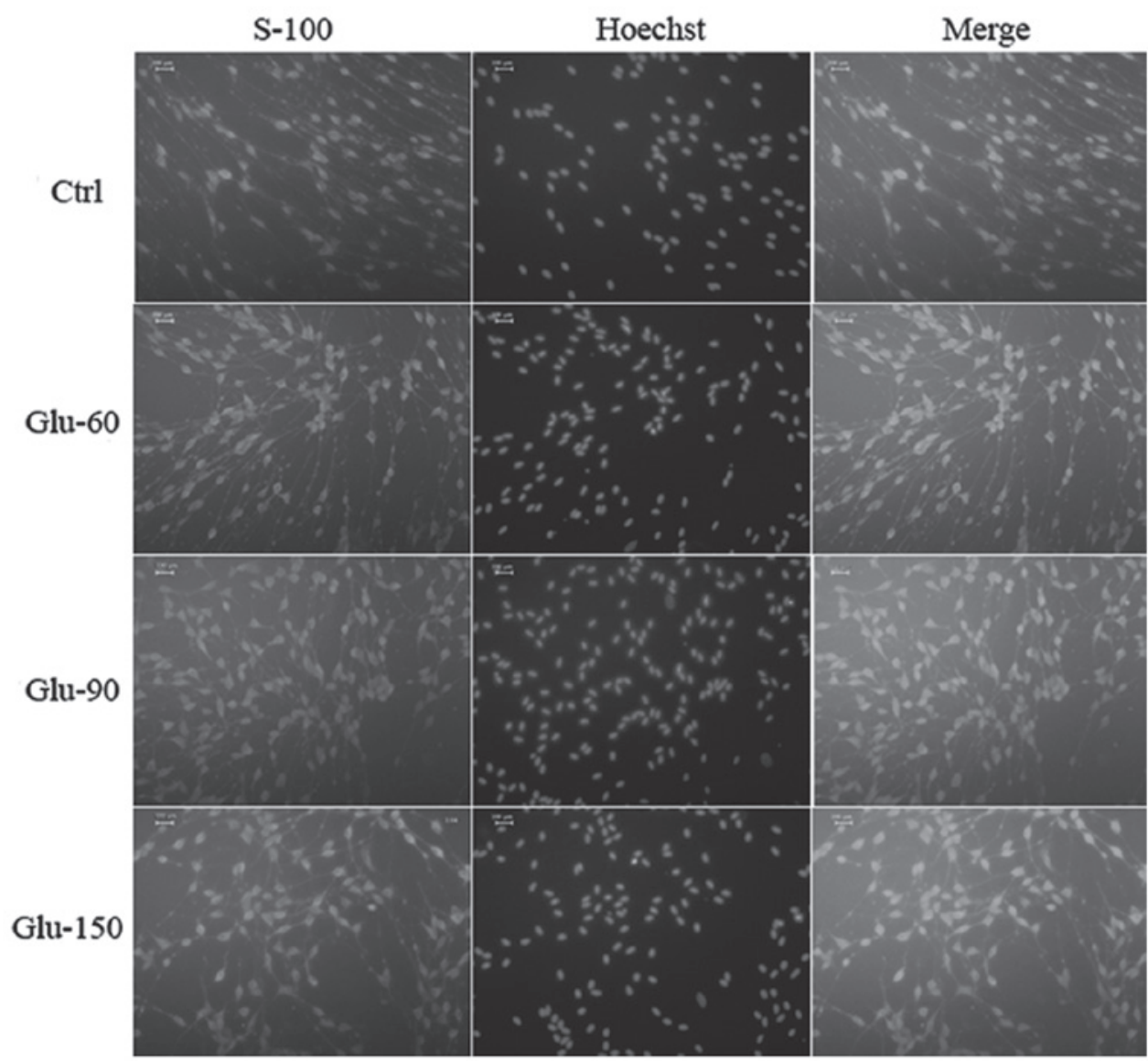

Figure 1. Morphological characteristics of SCs were evaluated by fluorescence microscopy. SCs were exposed to high glucose conditions for 24 h. S-100 was used to label SCs and Hoechst 33342 stained nuclei. SCs maintained their characteristic bipolar shape. Cell body size, process length and apoptosis rate in all groups were detected, and no abnormal characteristics were found. Bar, $100 \mu \mathrm{m}$. SCs, Schwann cells; Ctrl, control; Glu, glutatime (mM).

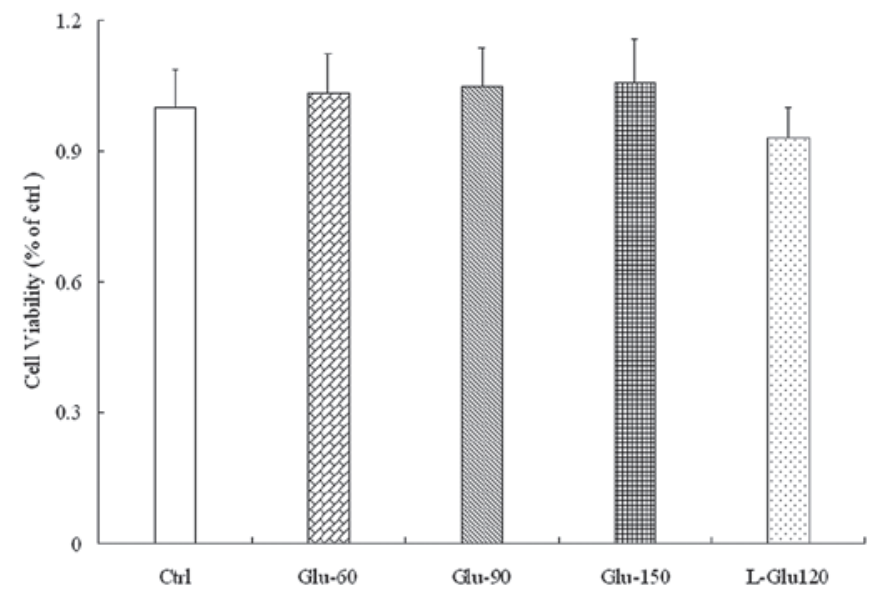

Figure 2. SC viability was examined using an MTT assay. SCs were exposed to high glucose conditions for $24 \mathrm{~h}$. There were no significant differences among the groups. High glucose levels did not reduce SC viability. Data were collected from 3 independent experiments and are expressed as the means \pm SEM. SC, Schwann cell; SEM, standard error of the mean. Ctrl, control; Glu, glutatime (mM).

protein levels using western blot analysis. SC-derived NGF and BDNF were significantly increased at glucose concentrations of $60 \mathrm{mM}(\mathrm{P}<0.05)$ and $90 \mathrm{mM}(\mathrm{P}<0.01)$ compared to the control cultures (Fig. 4). Additionally, NGF was decreased in cultures containing $150 \mathrm{mM}$ glucose compared to the control untreated cells $(\mathrm{P}<0.05)$, and there was no significant difference in BDNF levels between these cultures (Fig. 4).

High glucose levels activate p44/42 MAPK in SCs in a dose-dependent manner. MAPKs are a family of serine/ threonine-specific kinases that transduce extracellular stimuli to altered protein expression and have been shown to play a role in diverse cellular events ranging from proliferation and differentiation to apoptosis. To evaluate whether high glucose concentrations affect p44/42 MAPK (ERK1/2) activity, p44/42 phosphorylation was examined by western blot analysis. This demonstrated that high glucose concentrations significantly induced p44/42 MAPK activation in SCs in a dose-dependent manner (Fig. 4).

High glucose-induced SC expression of NTFs may be associated with the phosphorylation of p42/p44 MAPK. PD98059 effectively inhibits p42/p44 MAPK activation. Therefore, SCs were pre-treated with PD98059 for $2 \mathrm{~h}$ before they were incubated in serum-free medium containing $90 \mathrm{mM}$ glucose. Western blot analysis demonstrated that PD98059 prevented high glucose-induced p42/p44 MAPK phosphorylation in SCs. In addition, PD98059 further promoted the expression of NGF and BDNF in SCs cultured under high glucose conditions (Fig. 4). Our results suggest that the phosphorylation levels 
A
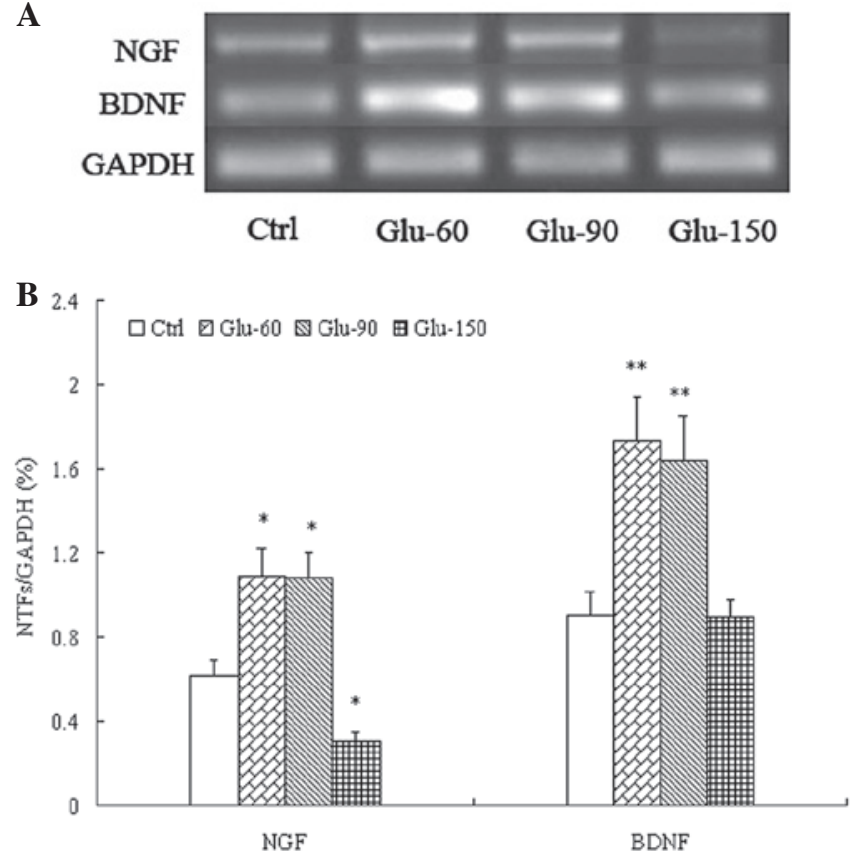

Figure 3. (A and B) NTFs mRNA levels in SCs were examined using RT-PCR analysis. SCs were exposed to high glucose conditions for $24 \mathrm{~h}$. High glucose levels elevated the mRNA levels of NGF and BDNF in SCs when glucose concentrations were in a certain range. Values of densitometric analyses are the means \pm SEM of 3 independent experiments. ${ }^{*} \mathrm{P}<0.05$ and ${ }^{* *} \mathrm{P}<0.01$ vs control group. NTFs, neurotrophic factors; SCs, Schwann cells; NGF, nerve growth factor; BDNF, brain-derived neurotrophic factor; SEM, standard error of the mean; Ctrl, control. Glu, glutamine (mM).

of p42/p44 MAPK may be associated with the expression of NTFs, and that the excessive activation of p42/p44 MAPK may, in turn, inhibit the expression of NTFs.

\section{Discussion}

In the current study, neither morphological abnormalities nor decreased viability were observed in SCs treated with up to $150 \mathrm{mM}$ glucose for $24 \mathrm{~h}$. Moreover, our results demonstrate that high glucose levels augment the expression of NGF and BDNF in cultured SCs in a short period of time. Our results are similar to the findings reported by Perrone et al (8) and Gumy et al (9). The fact that exposure to high glucose levels inhibited S100-induced SC morphological changes suggests that such changes do not occur under a high glucose environment (8). Furthermore, SCs isolated from neonatal rats did not undergo apoptosis after being exposed to $60 \mathrm{mM}$ glucose for $24 \mathrm{~h}$, and there was no detectable loss of cytochrome-c expression from the mitochondria, active caspase-3 or nuclear fragmentation (9).

Although the detailed pathogenesis remains unclear, both metabolic alterations in the cellular components (mainly neurons and SCs) and microvascular abnormalities are thought to play major roles in the development of diabetic neuropathy (10). Hyperglycemia-induced metabolic alterations include increased polyol pathway activity, enhanced nonenzymatic glycation, altered protein kinase $\mathrm{C}$ (PKC) activities and increased oxidative stress in the cellular components. Previously, certain studies have demonstrated that high glucose concentrations not only reduce NGF secretion (6) but also suppress the neurotrophin-3-induced production of NGF by

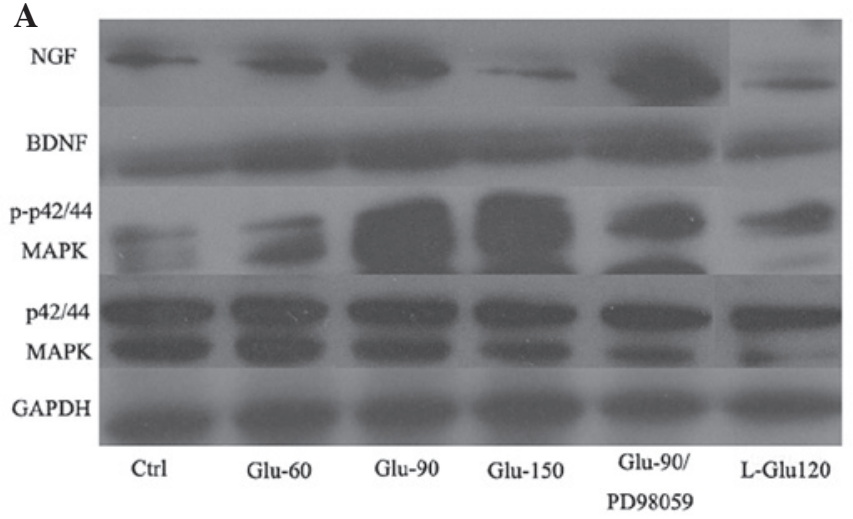

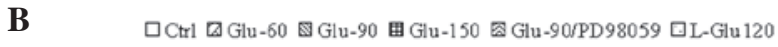

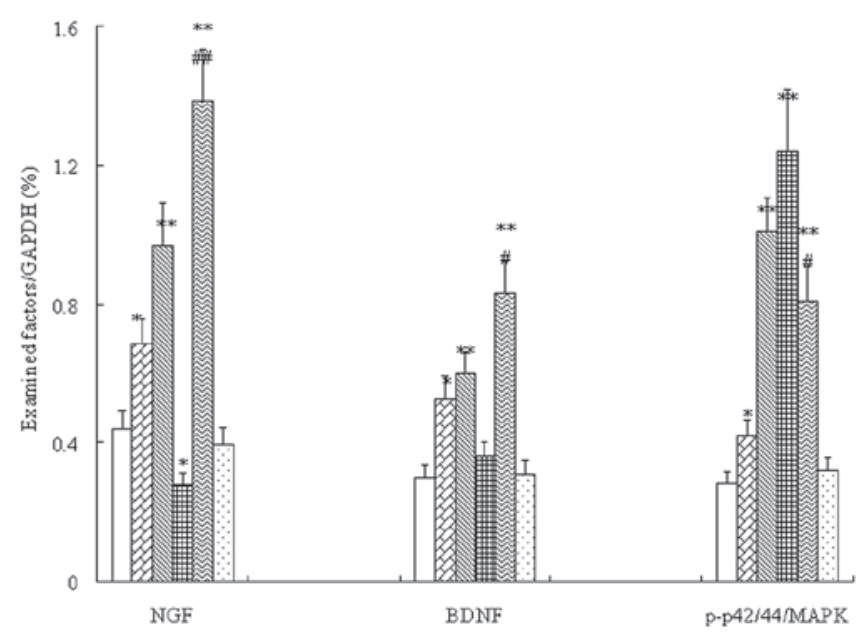

Figure 4. (A and B) Expression of SC-derived NTFs and p44/42 MAPK activity were detected by western blot analysis. SCs were exposed to high glucose conditions for $24 \mathrm{~h}$. Additionally, SCs were pretreated with PD98059 for $2 \mathrm{~h}$ before cells were incubated in serum-free medium containing $90 \mathrm{mM}$ glucose. High glucose reinforced the expression of NGF and BDNF in SCs within a certain range of glucose concentrations, induced p44/42 MAPK activation in a dose-dependent manner, and PD98059 prevented high glucose-induced p42/p44 MAPK phosphorylation in SCs and further promoted the expression of NGF and BDNF in the SCs exposed to high glucose levels. Values of densitometric analysis are the means \pm SEM of 3 independent experiments. ${ }^{*} \mathrm{P}<0.05$ and ${ }^{* *} \mathrm{P}<0.01$ vs. control group, ${ }^{\#} \mathrm{P}<0.05$ and ${ }^{\#} \mathrm{P}<0.01$ vs. Glu-90 group. SCs, Schwann cells; NGF, nerve growth factor; BDNF, brain-derived neurotrophic factor; SEM, standard error of the mean; MAPK, mitogen-activated protein kinase; Ctrl, control; Glu, glutamine (mM).

SCs (11). In our previous study, we also showed that SCs incur injury or apoptosis following 3 days of exposure to high glucose conditions (12). However, these changes were revealed in SCs exposed to high glucose conditions for a long period of time or in models of DPN. In the process of hyperglycemia, SCs experience a series of changes, including reactive, degenerative and proliferative changes. Reactive changes include accumulation of lipid droplets, pi granules of Reich and glycogen granules, increased numbers of subplasmalemmal vesicles, cytoplasmic expansion and capping (13). In the early glycemic status, due to the low number of mitochondria, high basal antioxidant enzyme expression and further increases in these enzymes in hyperglycemic SCs, SCs are not vulnerable to oxidative stress. Any significant increases in superoxide production in SCs were not able to be detected after up to 6 days of hyperglycemia (3). 
Increases in glucose levels are capable of inducing acute mitochondrial oxidative stress in the dorsal root ganglion (DRG) neurons, the sensory neurons normally impacted in diabetic neuropathy, whereas SCs are largely unaffected (14).

In addition, increases in NGF and BDNF in SCs exposed to high glucose levels were similar to the marked increases in the non-neuronal cells of the transected nerves (15) or in hypoxia/ reoxygenation-induced SCs (16). NTFs have long been proven to exert important functions in the protection of injured neurons and glial cells. NGF and BDNF are both potent NTFs. In the early response to hyperglycemia, $\mathrm{SC}$ is likely to promote self-protection and neuroprotection against hyperglycemiainduced damage through the expression of NGF and BDNF. NTFs interact with TrkA, TrkB and p75. NTFs activate Trk tyrosine kinases that signal via a ras-dependent pathway leading to the activation of the MAPKs and also through other enzymes, such as phosphatidylinositol-3 kinase, to promote neurocyte survival and nerve regeneration (17).

MAPKs are a family of serine/threonine-specific kinases that transduce extracellular stimuli to altered protein expression and have been shown to play a role in diverse cellular events ranging from proliferation and differentiation to apoptosis. Agthong et al (18) reported that the higher phosphorylation of ERK was observed in the proximal and distal nerve stumps compared to the contralateral intact nerve from one day to one month after crush injury occurred; the activation of ERK was mainly localized in the axons of the proximal segments; in the distal segments, however, active ERK was predominantly found in SCs forming Bungner's bands. Phospho-ERK1/2 levels rose rapidly in the distal stump following transection and remained high until at least 16 days later, and activation was delayed at sites distal to the transection site compared with segments adjacent to the cut site itself (19). The study by Meyer et al demonstrated that there were marked increases in NGF and BDNF mRNA in the non-neuronal cells of the transected nerve (15). These studies suggest that ERK1/2 activation may be involved in the expression of NTFs in SCs. Our results demonstrate that high glucose levels induce p42/p44 MAPK (ERK1/2) activation in a dose-dependent manner, and that phosphorylation levels of p42/p44 MAPK may be associated with the expression of NTFs in SCs. In addition, our findings support the theory that excessive activation of p42/p44 MAPK can inhibit the expression of NTFs. Detectable levels of activated ERK1/2 are found in wild-type nerves (20). However, high levels of the p42/p44 MAPK activation negatively regulate myelination (19). Since p42/p44 MAPK is substantially activated in diabetic nerves (21) and the expression of a constitutively active MAPK kinase 1 in SCs increases the phosphorylation of p42/p44 MAPK, this is sufficient to inhibit the forskolin-induced upregulation of myelin-associated glycoprotein, a myelin marker protein (22). Consistent with these results, the tamoxifen-induced activation of raf-1 (MAPK kinase kinase) in myelinated SC/sensory neuron co-cultures also increased SC dedifferentiation and the loss of myelin proteins (23).

In conclusion, short-time exposure to high glucose concentrations does not cause abnormal morphological characteristics or decrease the viability of SCs. However, high glucose levels induce p42/p44 MAPK activation in a dose-dependent manner and enhance the expression of NGF and BDNF in cultured
SCs, which may be associated with the phosphorylation levels of p42/p44 MAPK. Our findings provide direct evidence that SCs increase the expression of NTFs in the early response to hyperglycemia. Further studies are in progress at our institute to further clarify the details of this mechanism.

\section{Acknowledgements}

This study was supported by the Shanghai Leading Academic Discipline Project (No. S30201, S30205), the Shanghai Science and Technology Foundation of China (No. 1052nm04200), the International Cooperation projects of Shanghai Science and Technology Committee (No. 09410706200) and the Shanghai Education Committee.

\section{References}

1. Sango K, Suzuki T, Yanagisawa H, Takaku S, Hirooka H, Tamura M and Watabe K: High glucose-induced activation of the polyol pathway and changes of gene expression profiles in immortalized adult mouse Schwann cells IMS32. J Neurochem 98: 446-458, 2006

2. Feldman EL, Stevens MJ, Russell JW and Greene DA: Somatosensory neuropathy. In: Diabetes Mellitus. Porte DS, Sherwin RS and Baron A (eds). McGraw-Hill, New York, NY, pp771-788, 2003.

3. Zhang L, Yu C, Vasquez FE, Galeva N, Onyango I, Swerdlow RH and Dobrowsky R: Hyperglycemia alters the schwann cell mitochondrial proteome and decreases coupled respiration in the absence of superoxide production. J Proteome Res 9: 458-471, 2010.

4. Dobrowsky RT, Rouen S and Yu C: Altered neurotrophism in diabetic neuropathy: spelunking the caves of peripheral nerve. J Pharmacol Exp Ther 313: 485-491, 2005.

5. Yu C, Rouen S and Dobrowsky RT: Hyperglycemia and downregulation of caveolin-1 enhance neuregulin-induced demyelination. Glia 56: 877-887, 2008.

6. Tosaki T, Kamiya H, Yasuda Y, Naruse K, Kato K, Kozakae M, Nakamura N, Shibata T, Hamada Y, Nakashima E, Oiso Y and Nakamura J: Reduced NGF secretion by Schwann cells under the high glucose condition decreases neurite outgrowth of DRG neurons. Exp Neurol 213: 381-387, 2008.

7. Zhu H, Wang WJ, Ding WL, Li F and He J: Effect of panaxydol on hypoxia-induced cell death and expression and secretion of neurotrophic factors (NTFs) in hypoxic primary cultured schwann cells. Chem Biol Interact 174: 44-50, 2008.

8. Perrone L, Peluso G and Melone MA: RAGE recycles at the plasma membrane in S100B secretory vesicles and promotes schwann cells morphological changes. J Cell Physiol 217: 60-71, 2008.

9. Gumy LF, Bampton ET and Tolkovsky AM: Hyperglycaemia inhibits schwann cell proliferation and migration and restricts regeneration of axons and schwann cells from adult murine DRG. Mol Cell Neurosci 37: 298-311, 2008.

10. Mizisin AP and Powell HC: Pathogenesis and pathology of diabetic neuropathy. In: Textbook of Diabetic Neuropathy. Gries FA, Cameron NE, Low PA and Ziegler D (eds). Georg Thieme Verlag, New York, NY, pp83-169, 2003.

11. Suzuki T, Sekido H, Kato N, Nakayama Y and Yabe-Nishimura C: Neurotrophin-3-induced production of nerve growth factor is suppressed in Schwann cells exposed to high glucose: involvement of the polyol pathway. J Neurochem 91: 1430-1438, 2004.

12. Le Y, Zhu H, Wang WJ and Ding WL: Effects and mechanism of high-glucose on growth and apoptosis of Schwann cells in vitro. Acta Anatomica Sinica 40: 246-249, 2009.

13. Kalichman MW, Powell HC and Mizisin AP: Reactive, degenerative, and proliferative Schwann cell responses in experimental galactose and human diabetic neuropathy. Acta Neuropathol 95: 47-56, 1998.

14. Vincent AM, Kato K, McLean LL, Soules ME and Feldman EL: Sensory neurons and schwann cells respond to oxidative stress by increasing antioxidant defense mechanisms. Antioxid Redox Signal 11: 425-438, 2009. 
15. Meyer M, Matsuoka I, Wetmore C, Olson L and Thoenen $\mathrm{H}$ : Enhanced synthesis of brain-derived neurotrophic factor in the lesioned peripheral nerve: different mechanisms are responsible for the regulation of BDNF and NGF mRNA. J Cell Biol 119: 45-54, 1992.

16. Zhu H, Li F, Yu WJ, Wang WJ, Liu L, Wan LD, Le Y and Ding WL: Effect of hypoxia/reoxygenation on cell viability and expression and secretion of neurotrophic factors (NTFs) in primary cultured schwann cells. Anat Rec 293: 865-870, 2010.

17. Yoon SO, Casaccia-Bonnefil P, Carter B and Chao MV: Competitive signaling between TrkA and p75 nerve growth factor receptors determines cell survival. J. Neurosci 18: 3273-3281, 1998.

18. Agthong S, Kaewsema A, Tanomsridejchai $\mathrm{N}$ and Chentanez V: Activation of MAPK ERK in peripheral nerve after injury. BMC Neurosci 7: 45, 2006.

19. Jessen KR and Mirsky R: Negative regulation of myelination: relevance for development, injury, and demyelinating disease. Glia 56: 1552-1565, 2008
20. Fischer S, Weishaupt A, Troppmair J and Martini R: Increase of MCP-1 (CCL2) in myelin mutant schwann cells is mediated by MEK-ERK signaling pathway. Glia 56: 836-843, 2008.

21. Purves TD, Middlemas A, Agthong S, Jude EB, Boulton AJM, Fernyhough $\mathrm{P}$ and Tomlinson DR: A role for mitogen-activated protein kinases in the etiology of diabetic neuropathy. FASEB J 15: 2508-2514, 2001.

22. Ogata T, Iijima S, Hoshikawa S, Miura T, Yamamoto S, Oda H, Nakamura K and Tanaka S: Opposing extracellular signal-regulated kinase and Akt pathways control schwann cell myelination. J Neurosci 24: 6724-6732, 2004.

23. Harrisingh MC, Perez-Nadales E, Parkinson DB, Malcolm DS, Mudge AW and Lloyd AC: The Ras/Raf/ERK signalling pathway drives Schwann cell dedifferentiation. EMBO J 23: 3061-3071, 2004. 